\title{
SKiN
}

\section{Designing pseudo-physiology into personal technology devices to create enhanced interactions through visceral signifiers and behaviours}

\author{
Mr. Andrew O'Dowd \\ Department of Design, Photography and Advertising \\ Edinburgh Napier University, Edinburgh, UK \\ a.odowd@napier.ac.uk
}

\begin{abstract}
The majority of technology is now personal. Humans, in turn, are expected to and have personal relationships with their devices. The issue is that humans are biological beings and relate to the world as animals, in a biological way. In contrast, personal technologies are increasingly losing any symbol of visceral behaviour or form. As a language, gestural interactions and visceral behaviours of objects are more capable of immediately communicating emotional intent and response than constructed oral language.
\end{abstract}

Keywords-interaction; affordance; behaviour; object; skin; surface;

\section{SIGNIFICANCE OF THE RESEARCH}

The current epoch we live in is a society consumed by small, smooth, nearly buttonless devices. Through these objects humans are expected to achieve both emotional and functional interactions with the world around them. And to some extent they do. The world we live in is ultra-functional and still the interactions we have with the world through our connected objects attempt to do so with intuition and personal consideration. Here we are living with objects and devices that we have formed relationships with, therefore to discuss design and emotion without referring to Donald Norman's work would be naive. The moment a designer begins to consider form and function, in whichever order, they are engaging with a process that Norman has discussed in great depth since the 1980 s.

The purpose of this investigation is not to recite the thinkings of Norman but to explore the current state of society in relation to the interactions we have with the designed things Norman presents a platform from which to begin. In his 2004 book Emotional Design, Norman proposes that without emotions, your decision-making ability would be impaired [1]. To consider this from a users perspective it would suggest that any decision for or against interactions with objects uses subconscious emotional instructions that must and will incorporate some level of visceral, emotional action. This proposition is also considered by Evans [2] who's research into emotion reveals that the universality of basic emotions argues strongly for their biological nature. These emotions allow us to evaluate many things including potential response of an object, our desire for that response and our willingness to obtain an unsolicited response also.

Norman's categorisation of the interactions people have with objects into 3 parts, visceral, behavioural and reflective go a long way towards demonstrating the physiological way in which humans approach the world. The gap which has appeared in this proposal is not in their definition but in the manner in which the designed world has now interfered with the order of them. Norman discusses visceral design as the experiences felt upon being presented with a designed object. As he states, visceral is about initial reactions [3].

Considering this from a more human perspective Schulkin presents the argument that the mind and body (the behavioural and the visceral) are a collaborative system. He identifies, there must be some trade off between novelty and familiarity in aesthetic judgments [4]. When we observe the trend of personal device design over the past decade there is a growing effect. Humans expect objects to be nondescript, void of visceral attributes, of muted or monochromatic colour, and explicit in their instructions for interactivity. This as Norman discusses is behavioural design. The gap which I mention previously is occurring at this place. If humans have evolved over millions of years to first evaluate the potential risk and reward of interaction with the wider world and we are now designing a world devoid of any visceral signal then surely we are moving toward a world where behavioural design is a consideration human users will engage with more quickly than visceral design.

\section{AUDIENCE}

The audience for this demo is primarily practitioners and academics in the fields of user-object interaction design, interface design, experiential design and consumer product design.

\section{ROUGH AGENDA FOR DEMO}

The demo is intended to open up discussion around the topic of trends in personal device technology design. This will be attempted through both a live demonstration of the SKiN experiment and presentation of observations and findings from initial research activities. 
Running order:

- Introduction of topic

- Background of experiment

- Observations (Video and Photographic) from research

- Demonstration of experiment

- Q \& A \& Feedback.

\section{PRESENTER BACKGROUND}

My work is in the area of product and spatial interaction design. I have an academic and practice interest in how humans behave and respond to technological enhanced objects and spaces. My background is in Product Design Engineering and Tangible Interaction Design. I have worked as a Lecturer in Design for four years during which time I have been involved in a number of projects ranging from a trans-media theatre design at the Edinburgh International Festival 2013, interactive \& immersive installation design in a collaborative project with the Zhengzhou University of Light Industry in China, and emotional object design through student design projects at both undergraduate and postgraduate level. My specific areas of interest are in responsive objects and spaces, emotionally enhanced objects and spaces, decay and error in digital systems, design research methods, ethnography, transhuman and trans-object design.

\section{EXPERIMENTATION}

The organic visceral world is a confusing one. Full of risk, full of potential for misuse and error. Technology design has almost removed this attribute from our lives. If not almost, than it is certainly attempting to. Exact responsive function and pseudo-cognitive computing are the mantras of design today. As design continues to put behavioural design before visceral then the opportunity for humans to develop personal, emotional, relationships with their connected objects will soon reach a plateau.

In order to conduct a primary investigation into this trend a number of experiments were created to observe the various interactions humans have with objects based upon their current responsive ability and then with enhance responsive ability.

Developing on the Heider and Simmel research on Apparent Behaviour, the experiment intended to observe how biological, cognitive and intelligence characteristics can be attributed to objects which are themselves not biological but exhibit behaviours akin to biological organisms. Their findings inferred that a characteristic feature of this organization in terms of actions is the attribution of the origin of movements to figural units and to motives [5]. From their findings there is suggestion that the speed of response of third part 'actors' determines their intent as perceived by the subject. Drawing on this conclusion this experiment will primarily focus on the visceral responsiveness of the skin of a technology in suggesting a biological awareness and a status of emotion.

\section{A/V AND COMPUTER REQUIREMENTS}

The demo will primarily be a presentation of the current and developing research for that reason there will be minimal materials provided to attendees. The demo does intend to allow attendee's to experiment and interact with the artefacts of the research. Depending on number of attendees the demo may require a projector in order to present the detailed elements of the experiments. Current developments of the research are in the area of visceral interpretation through digital avatar generation. Should this aspect of the research be ready to demonstration then a wireless internet connection will also be required.

\section{RELATING TO OBJECTS}

The visceral way in which humans engage with the world is full of subtlety. Colours, textures, specific movements, and sounds are among the signifiers that provide humans with the information they need to evaluate an objects characteristics (e.g. way of interaction, function or responsive behaviour). In the designed world there are many examples of objects which drawn upon these characteristics to denote their attribute. Door handles for example in association to the surface they are connected to and the height they are positioned communicate to us how to engage with them and their potential. So too a kettle, proposes it's function as a vessel for liquid and with a singular switch to interact with, human users are left with little confusion of how to initiate their function.

In 1986, Gibson proposed that what we perceive when we look at objects are their affordances. That these affordances inform human users of the qualities of the object i.e. it's purpose, how to use it, and how to know when we are using it correctly. In contrast, when we look at the visceral design trend of current personal device technologies there is a distinct absence of these affordances. A singular button will propose where to begin functional interaction but immediately the visceral design concludes and behavioural or functional design commences.

The surfaces of the world we now live in have diluted the visceral in place for rapid functionality. In doing so the opportunity for human users to develop visceral relationships with their personal technologies is greatly diminished. We can compare this kind of relationship to the ones humans have with other animals. As Gibson [6] states, behavior affords behavior, meaning that humans have evolved over millions of years to respond and act on the world as a dialogue rather than an instructor. If a dog growls at you, you modify your behaviour accordingly. Fail to do that and your desired interaction with the animal will not be so likely. In this same approach, personal technology devices which present no visceral behaviour will fail to achieve the elementary principle of animal interaction. Failing this results in the potential interaction, and in turn the object, falling into a category outside of intelligent.

\section{REFERENCES}

1. D. Norman, "Emotional Design," Basic Books: New York, 2004, pp.10.

2. D. Evans, "Emotion: A Very Short Introduction," Oxford University Press: Oxford, 2001, pp.13.

3. D. Norman, "Emotional Design," Basic Books: New York, 2004, pp.64.

4. J. Schulkin, "Bodily Sensibility," Oxford University Press: Oxford, 2004, pp.64. 
Computing Conference 2017

18-20 July 2017 | London, UK

5. F. Heider \& M. Simmel, "An Experimental Study of Apparent Behavior," The American Journal of Psychology, Vol. 57, No. 2, April 1944, pp. 243-259
6. J. Gibson, "The Ecological Approach to Visual Perception," Routledge: London, 1986. 